\title{
Broadband observations of GRB 110731A with Fermi, Swift, GROND and MOA
}

\author{
Johan Bregeon ${ }^{* \dagger}$ \\ INFN Sez. di Pisa, Largo Pontecorvo, 3, 56124 Pisa (PI), Italy \\ E-mail: johan.bregeon@pi.infn.it
}

We report on the multi-wavelength observations of the bright long GRB 110731A detected by Fermi and Swift, and follow-up observations by the MOA and GROND optical telescopes. The analysis of the prompt phase reveals that this GRB shares many features with the other bright bursts observed by the Fermi-LAT during its first 3 years on-orbit. The wealth of multiwavelength observations allowed temporal and spectral analyses in different epochs, favoring emission from the forward shock in a wind-type medium. The temporally-extended emission observed by the LAT is most likely the highest-energy extension of the afterglow. Both the singlezone pair transparency constraint for the prompt signal, and the spectral and temporal analysis of the forward shock afterglow emission, independently lead to an estimated bulk Lorentz factor of the jet $\Gamma \sim 500-550$.

Gamma-Ray Bursts 2012 Conference - GRB2012,

May 07-11, 2012

Munich, Germany

\footnotetext{
* Speaker.

${ }^{\dagger}$ Thanks to hard work by David Gruber, Daniel Kocevski, Soebur Razzaque, Eleonora Troja, Giacomo Vianello, to Fermi and Swift collaborations, and to the GROND and MOA groups for sharing their data.
} 
Although Swift and Fermi have been taking data together in the past 3 years, only 4 bursts to date, have benefited from joined Swift/BAT and Fermi/LAT detection. The first three are: GRB 090510, a very bright short GRB for which the afterglow was observed contemporaneously [1], GRB 100728A, a very long burst with GeV extended emission until $850 \mathrm{~s}$ and a series of strong X-ray flares, and GRB 110625A, a joint BAT/GBM trigger, with XRT detection simultaneous with LAT, but with no significant LAT prompt emission. The fourth one is GRB 110731A, a bright and long GRB with GeV prompt and extended emission, the first to benefit from a full joint coverage from Fermi and Swift from the burst trigger time and for more than 30 minutes. Our GRB 110731A data were completed with ground-based observations by the Gamma-ray Burst Optical/NearInfrared Detector (GROND) and the Microlensing Observations in Astrophysics (MOA) teams.

Throughout the paper, the GBM trigger time is our reference $T_{0}$, and the convention $F_{v, t} \propto$ $v^{-\beta} t^{-\alpha}$ has been followed, where the energy index $\beta$ is related to the photon index $\Gamma=\beta+1$.

\section{Observations}

On 2011 July 31 at 11:09:29.94 (UT), GBM triggered on GRB 110731A which caused an Autonomous Repoint Request (ARR) for high peak flux. GRB 110731A was already well within the LAT field of view so that Fermi could take excellent quality data of the prompt and extended emissions up to 2.5 hours after the burst trigger. GRB 110731A triggered the Swift/BAT at 11:09:30.45 UT. Swift slewed immediately to the burst, and its narrow-field instruments, XRT and UVOT, began observations $56 \mathrm{~s}$ after the BAT trigger. An accurate afterglow position was rapidly determined by the UVOT at $\mathrm{RA}=18^{\mathrm{h}} 42^{\mathrm{m}} 00.99^{\mathrm{s}}, \mathrm{DEC}=-28^{\circ} 32^{\prime} 13.8^{\prime \prime}$ (J2000, [2]), with an error radius of $0.5 \operatorname{arcsec}$ (90\% confidence).

The MOA telescope quickly reacted to the GCN alert, started observations $3.3 \mathrm{~min}$ after the Swift/BAT trigger and took several $30 \mathrm{~s}$ images for the next $105 \mathrm{~min}$ in I and V bands. Due to bad weather, GROND was only able to provide late time, but deep, observations $2.7 \mathrm{~d}$ after the trigger in the $g^{\prime} r^{\prime} i^{\prime} z^{\prime}$ and $J H K$ bands.

\section{Prompt emission results}

Figure 1, left, shows the multi-wavelenght light curve for the GBM and LAT observations of the prompt phase. GRB 110731A is a quite standard bright long burst $\left(\mathrm{T}_{90, \mathrm{GBM}, 50-300 \mathrm{keV}}=7.3 \pm\right.$ $0.3 \mathrm{~s}$ ) that presents many of the common features of most LAT bursts: high variability, with a peak in flux observed in all energy bands, a delayed on-set of the $>100 \mathrm{MeV}$ emission $\left(\mathrm{T}_{05, \mathrm{LAT}} \sim 2.44 \mathrm{~s}\right)$ that also last longer $\left(\mathrm{T}_{90, \mathrm{LAT}}=14.33_{+16.79}^{-2.55} \mathrm{~s}\right)$ than the low energy emission. The typical variability time scale of the burst was studied following [3]: we estimated the median value for the full width half maximum to be $0.428 \pm 0.025 \mathrm{~s}$, in the $8 \mathrm{keV}-1 \mathrm{MeV}$ range. The highest photon energy during the prompt phase is a $2.0 \mathrm{GeV}$ event at $T_{0}+8.270 \mathrm{~s}$, having a probability of $10^{-6}$ to be associated to background.

For the spectral analysis we followed the standard procedure described for instance in [4], using LAT P7TRANSIENT_V6 class data in a joint fit with the GBM data from detectors NaI 0 and 3, and BGO detector 0. The fits have been done with XSPEC using the PG-statistic [5], and we 
cross-check the results with rmfit $^{1}$. We first studied the time integrated spectrum from $\mathrm{T}_{05, \mathrm{LAT}}$ to $\mathrm{T}_{90, \text { GBM }}$, i.e. $\left[T_{0}+3.0 \mathrm{~s} ; T_{0}+7.56 \mathrm{~s}\right]$, which covers the time region with the maximum of signal in both detectors. In this time interval, the respective statistics and degrees of freedom for the fits are: 440.7/354 for the Band function, 405.5/352 for the Band plus power-law, and 390/352 for a Band plus power-law plus cutoff. Our preferred model is a Band function $\left(\alpha_{\text {Band }}=0.03_{-0.12}^{+0.15}\right.$, $\beta_{\text {Band }}=-2.40_{-0.20}^{+0.10}, E_{0}=349_{-28}^{+31} \mathrm{keV}$ for $E_{\text {peak }} \sim 710 \mathrm{keV}$ ), plus a power-law with photon index $\gamma=-1.96_{-0.05}^{+0.09}$ : adding the power-law extra-component to the Band function improves the fit goodness by more than $5.5 \sigma$. The count spectrum, forward folded Band plus power-law model and fit residuals are shown on the right of figure 1. Adding a cutoff to the power-law leads to a further improvement of the fit by $\sim 4 \sigma$ : while not being formally statistically required, we consider this cutoff at $E_{\text {cutoff }}=390_{-120}^{+220} \mathrm{MeV}$ significant enough to be mentioned. We then ran the spectral analysis on a set of time intervals determined by running a Bayesian block analysis [6] on the LAT low energy events light curves: these are intervals $a, b, c, d$, with boundaries $T_{0}+$ $(0,2.44,5.44,6.06,8.52) \mathrm{s}$ and are highlighted in Fig. 1. In interval $a$, in which there is basically no LAT signal, the best fit is obtained for a Comptonized model (a power-law with an exponential cutoff). In all other time intervals $b, c, d$, the best fit is obtained with a Band function, with $\beta \sim-2.2--2.3$, and the extra-component measured on the time integrated spectrum seems to be present but not very significant $\sim 3 \sigma$.
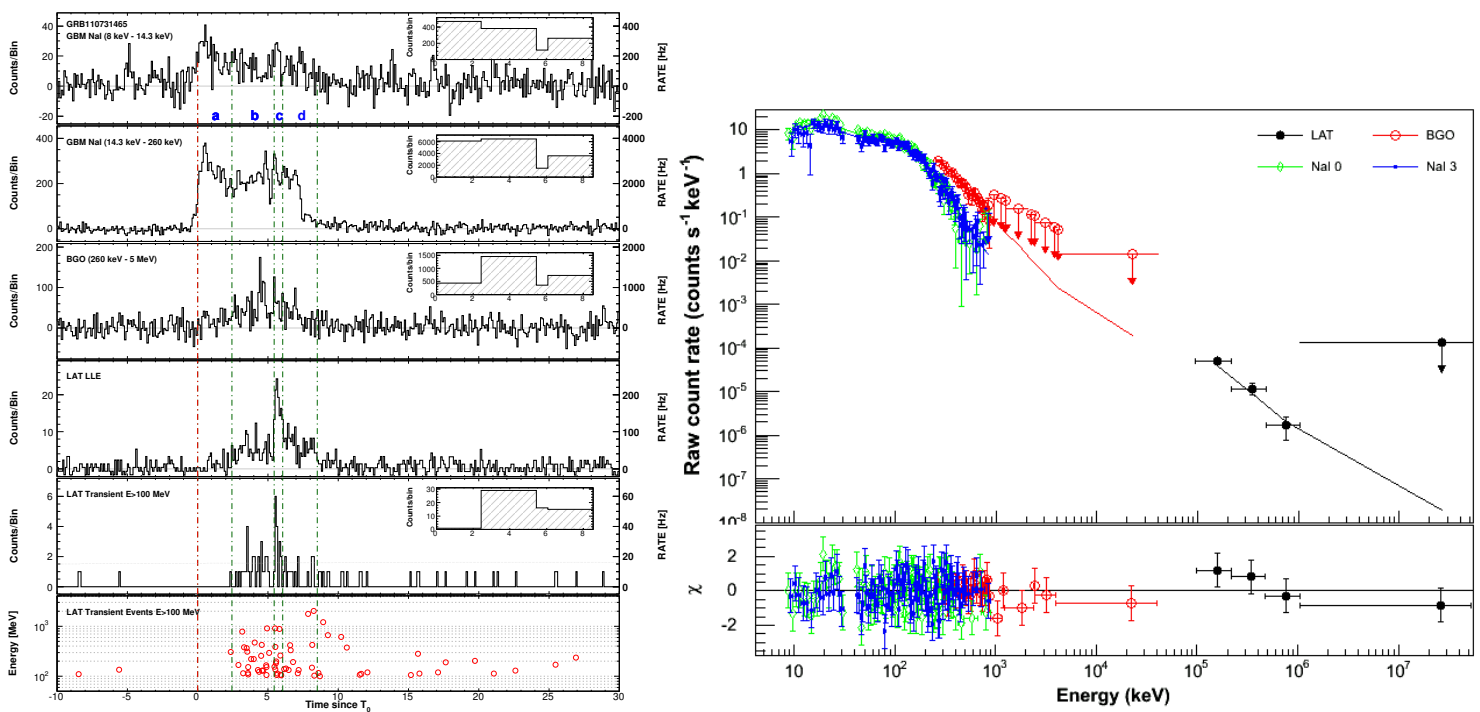

Figure 1: Left: Prompt phase light curve for the GBM and the LAT. Right: Joint spectral fitting of GBM and LAT data for the time integrated spectrum. Count spectra (points) and best-fit (BAND+PL) model (lines) on the top, and residuals at the bottom.

\section{Multi-wavelength afterglow study: light curves and spectral energy densities}

Figure 2 shows the wealth of quality data available for the study of the afterglow of GRB 110731A. The LAT extended emission was explored by running an unbinned likelihood analysis with the

\footnotetext{
${ }^{1}$ rmfit for GBM and LAT analysis was developed by the GBM Team and is publicly available at fermi.gsfc.nasa.gov/ssc/data/analysis/
} 
Fermi ScienceTools-v9r25p1 and P7TRANSIENT_V6 instrument response functions. The model used for the likelihood was composed of a single power-law component for the burst itself, the particle background template and a template model for the Galactic diffuse emission for which the normalization was fixed during the fit. After the peak flux at $T_{0}+5.5 \mathrm{~s}$, the LAT flux decay is well fit by a single power-law of index $\sim-1.5$, as is shown in figure 2 .

XRT light curves and spectra were extracted in the nominal $0.3-10 \mathrm{keV}$ energy range by applying standard screening criteria: note that the light curve in blue in figure 2, shows two $\mathrm{X}-$ ray flares, peaking at $T_{0}+70 \mathrm{~s}$ and $T_{0}+110 \mathrm{~s}$ respectively, that made the analysis slightly more complex. The $\mathrm{X}$-ray flux decay was better fit by a broken power-law with best fit parameters $\alpha_{X, 1}=1.10 \pm 0.02, \alpha_{X, 2}=1.32 \pm 0.03$ and $t_{b k}=4.6_{-1.6}^{+2.6} \mathrm{ks}$.

The optical data comprise the UVOT, MOA and GROND data. In order to better constrain the optical temporal decay we created a single light curve (see Fig. 2) from all the UVOT filters by re-normalizing each light curve to the $V$-band [7], using flux conversion factors from [8]. The MOA data in $I$ and $V$ bands have been reduced following a standard procedure, normalized to the GROND filter system and the GROND late time observations were additionally used to fix the zero point and set the systematic uncertainties. The GROND field was calibrated against an SDSS standard star field observed just before the beginning of the first epoch observations under photometric conditions. All the optical data points were fit together with a single power-law, which provides a good fit for index $\alpha_{\mathrm{opt}}=1.37 \pm 0.03$.
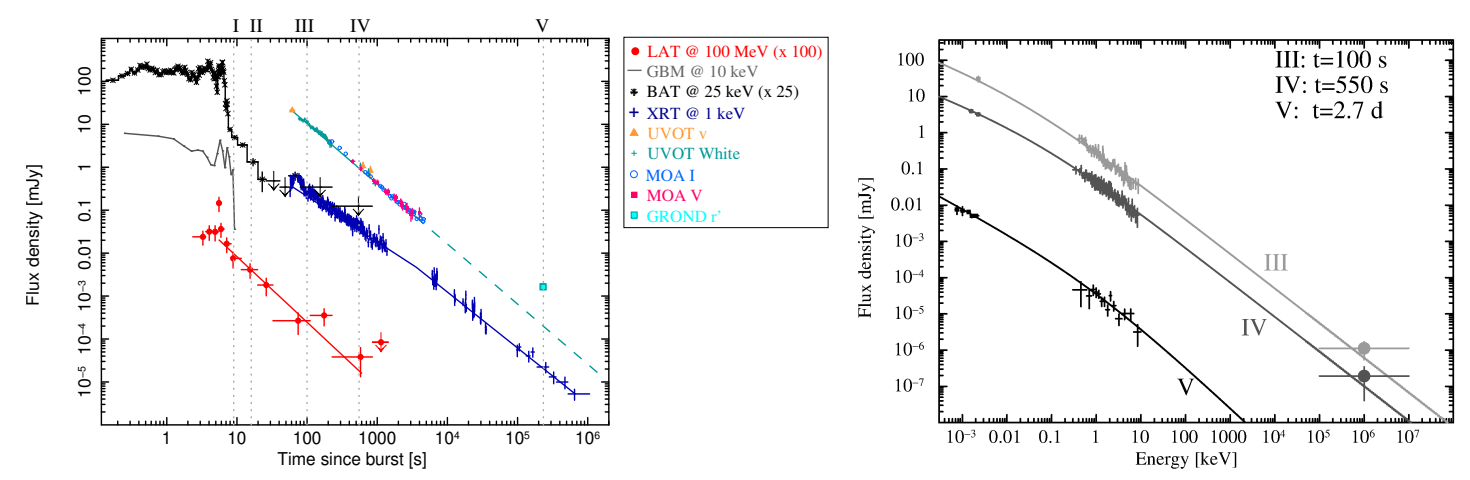

Figure 2: Left: Multi-wavelength light curves for the full observation period. Right: SED fits for the late time epochs III, IV and V

For the broadband spectral analysis of the afterglow, we had to define new time intervals in order to achieve a significant detection in the LAT energy band (epochs I-III), sufficient statistics in the X-ray spectrum (epoch IV) and to match GROND observations for epoch V: (I): $T_{0}+9 \mathrm{~s}$, (II): $T_{0}+16 \mathrm{~s}$, (III): $T_{0}+100 \mathrm{~s}$, (IV): $T_{0}+550 \mathrm{~s}$, and (V): $T_{0}+2.74 \mathrm{~d}$. Each SED was fit using $X S P E C$, with an absorbed power-law or an absorbed broken power-law, where the spectral slopes $\beta_{1}$ and $\beta_{2}$ were tied to $\beta_{2}=\beta_{1}-0.5$, as predicted by the closure relations for a cooling break. The Galactic $X$-ray absorption and reddening were fixed to the values of $\mathrm{N}_{\mathrm{H}}=1.0 \times 10^{21} \mathrm{~cm}^{-2}$ and $E(B-V)=0.18[2]$, respectively.

For early epochs I and II, the single power-law fit over 5 orders of magnitude is a very good representation of the data. The resulting photon indices are $\gamma_{\mathrm{I}}=1.87_{-0.11}^{+0.07}$ and $\gamma_{\mathrm{II}}=1.95_{-0.11}^{+0.07}$, and 
are compatible with the measured $X$-ray photon index $\Gamma_{X}$ at later times. We note however that the BAT flux decay index, which is $\alpha_{\mathrm{BAT}}=2.3 \pm 0.3$, is only barely consistent with the LAT flux decay $\alpha_{\mathrm{LAT}}=1.5 \pm 0.2$

For late time epochs IV and V, the single power-law fit gives a good description of the data. However, because of the flux decay behavior, in particular because the optical flux decays faster than the $\mathrm{X}$-ray flux, we decided to test a forward shock in a wind model via a broken power-law fit of both epochs together. We linked the optical and X-ray spectral index with $\beta_{o p t}=\beta_{X}-0.5$, imposed the break energy to grow with time $E_{\text {break }} \sim \sqrt{t}$, and tied the host extinction and absorption. The result is shown on the right side of figure 2 , including the crosscheck for epoch III. The parameters obtained for the best fit are: $\beta_{\text {opt }, I V}=0.45_{-0.07}^{+0.09}, \beta_{X, I V}=0.95_{-0.07}^{+0.09}, E_{\text {break }, I V}=0.04_{-0.01}^{+0.03} \mathrm{kev}$ for epoch IV, and $\beta_{\text {opt }, V}=0.66_{-0.03}^{+0.03}, \beta_{X, V}=1.16_{-0.03}^{+0.03}, E_{\text {break }, V} \sim 0.8 \mathrm{kev}$ for epoch $\mathrm{V}$.

\section{Interpretation and conclusions}

GRB $110731 \mathrm{~A}$ is very bright in the Fermi LAT energy range with an isotropic-equivalent gamma-ray $(10 \mathrm{keV}-10 \mathrm{GeV})$ energy release of $E_{\text {iso }} \sim 6 \times 10^{53}$ erg within the prompt phase of the first $\sim 8.5 \mathrm{~s}$. The analysis of the prompt phase has shown a few interesting features: the high energy emission is delayed by a couple of seconds with respect to the low energy emission, a strong extra power-law component grows through the burst, and the temporally extended $\mathrm{GeV}$ emission last for hundreds of seconds. These features are actually similar to other bright LAT bursts such as GRB 090510, GRB 090902B, GRB 090926A. In GRB 110731A we also found hints of a $\sim 4 \sigma$ cutoff at $\sim 400 \mathrm{MeV}$ in the extra power-law component: assuming that the cutoff is due to pair creation in the internals shocks with a variability time scale $\Delta t \sim 0.4 \mathrm{~s}$, and for a single zone steady state model with homogeneous photon distribution, we derive the bulk Lorentz factor of the jet to be $\Gamma_{j e t}=530 \pm 10$. Following the parametrization of [9], we also estimated $\Gamma_{\text {jet }}$ for a multi-zone model and obtained a lower limit of 300 .

The analysis of the afterglow has shown that for epochs I and II a single power-law fit from the $\mathrm{keV}$ to the $\mathrm{GeV}$ leads to a good representation of the data. In addition to this, the obtained photon index is compatible with the spectrum of the temporally extended $\mathrm{GeV}$ emission at a late time, therefore the LAT extended emission, as early as $T_{0}+8.3 \mathrm{~s}$ from the earliest broadband SED I, is compatible with the afterglow synchrotron emission in the fast-cooling regime. Epoch III is difficult to analyze because of its X-ray flares: it seems to be compatible with the time of the transition from the fast- to slow-cooling spectrum. Finally, during epochs IV and V, we notice that optical flux decays faster than the X-ray flux, and the fit to the data with a smoothly broken powerlaw fit with $\beta_{\text {opt }}=\beta_{X}-0.5$ and $E_{\text {break }} \sim \sqrt{t}$ lead to a set of parameters compatible with a wind environment and slow-cooling spectrum: $\beta_{X}=(2 / 3) \alpha_{X}+1 / 3, \beta_{\text {opt }}=(2 / 3) \alpha_{\text {opt }}-1 / 3, v_{\text {opt }}<$ $v_{c}<v_{X}$. In epoch $\mathrm{V}$, a steeper decrease of $\mathrm{X}$-ray flux is further explained by the cooling break, $v_{c} \sim \sqrt{t}$, approaching the $X$-ray band after $t_{\text {break }} \sim 4.6 \mathrm{ks}$. The afterglow modeling, following [10], lead to the following set of parameters: a low magnetic field $\varepsilon_{B} \sim 10^{-2}$, a low radiative efficiency $\varepsilon_{E} \sim 10^{-3}, 5$ times larger kinetic energy than gamma-ray energy, $E_{k} \sim 3 \times 10^{54} \mathrm{erg}$, and a large bulk Lorentz factor $\Gamma_{j e t} \sim 500$. It's worth noticing that the coasting bulk Lorentz factor $\Gamma_{j e t} \sim 500$ from the afterglow model is compatible with the value/lower limit obtained from the $\gamma \gamma$ opacity argument in the internal shocks of the prompt phase. 


\section{Acknowledgments}

The Fermi LAT Collaboration acknowledges support from a number of agencies and institutes for both development and the operation of the LAT as well as scientific data analysis. These include NASA and DOE in the United States, CEA/Irfu and IN2P3/CNRS in France, ASI and INFN in Italy, MEXT, KEK, and JAXA in Japan, and the K. A. Wallenberg Foundation, the Swedish Research Council and the National Space Board in Sweden. Additional support from INAF in Italy and CNES in France for science analysis during the operations phase is also gratefully acknowledged. The Fermi GBM collaboration acknowledges support for GBM development, operations and data analysis from NASA in the US and BMWi/DLR in Germany.

We gratefully acknowledge the contributions of dozens of members of the Fermi, Swift, GROND and MOA teams and our subcontractors who helped make these instruments possible. This work made use of data supplied by the UK Swift Science Data Centre at the University of Leicester.

Part of the funding for GROND (both hardware as well as personnel) was generously granted from the Leibniz-Prize to Prof. G. Hasinger (DFG grant HA 1850/28-1).

We acknowledge the MOA collaboration for TOO time, and University of Canterbury / Mt John Observatory for allowing MOA to use the B\&C telescope.

\section{References}

[1] De Pasquale et al., M. Swift and Fermi Observations of the Early Afterglow of the Short Gamma-Ray Burst 090510, ApJL, 709 (2010) L146 [arxiv: 0910 . 1629]

[2] Oates, S. R., Ukwatta, T., Sakamoto, T., et al., Swift Observations of GRB 110731A, GCN Report, 343.1 (2011)

[3] Bhat, P. N., Briggs, M. S., Connaughton et al., V., Temporal Deconvolution Study of Long and Short Gamma-Ray Burst Light Curves, ApJ, 744 (2012) 141 [arxiv:1109.4064B]

[4] Ackermann et al., M., Detection of a Spectral Break in the Extra Hard Component of GRB 090926A, ApJ, 729 (2011) 114 [arxiv:1101.2082]

[5] Arnaud, K., XSPEC: The First Ten Years, ASP Conf. Series, Vol. 101, Astronomical Data Analysis Software and Systems V, eds. Jacoby G. and Barnes J., 17 (1996)

[6] Scargle, J. D., Studies in Astronomical Time Series Analysis. V. Bayesian Blocks, a New Method to Analyze Structure in Photon Counting Data, ApJ, 504 (1998) 405

[7] Oates, S. R., Page, M. J., Schady, P., et al., A statistical study of gamma-ray burst afterglows measured by the Swift Ultraviolet Optical Telescope, MNRAS, 395 (2009) 490 [arxiv: 0901.3597 ]

[8] Breeveld, A. A., Landsman, W., Holland, S. T., et al., An Updated Ultraviolet Calibration for the Swift/UVOT, ASP Conf. Series, Vol. 1358, ed. J. E. McEnery, J. L. Racusin, \& N. Gehrels (2011) 373-376 [arxiv:1102.4717]

[9] Hascoët, R., Daigne, F., Mochkovitch, R., \& Vennin, V., Do Fermi Large Area Telescope observations imply very large Lorentz factors in gamma-ray burst outflows?, MNRAS, 421 (2012) 525 [arxiv:1107.5737]

[10] Granot, J., \& Sari, R., The Shape of Spectral Breaks in Gamma-Ray Burst Afterglows, ApJ, 568 (2002) 820 